\title{
Mirando al Futuro: Desafíos y Oportunidades Para el Desarrollo de los Adolescentes en Chile
}

\section{Towards the Future: Challenges and Opportunities for Adolescent Development in Chile}

\author{
M. Loreto Martínez Guzmán \\ Pontificia Universidad Católica de Chile
}

\begin{abstract}
El desarrollo adolescente depende de las fortalezas y vulnerabilidades de cada adolescente, como también de los contextos de vida en que ellos se desenvuelven. Este artículo amplía el foco de análisis desde lo individual a la comprensión de las características de los contextos de vida de los adolescentes, y de las oportunidades que estos ofrecen para desarrollar competencias y habilidades. Analiza dos desafíos para la formulación de políticas (inequidad social, fortalecimiento del capital social) y propone reemplazar el énfasis en las conductas de riesgo por la promoción de la competencia en ellos. Enfatiza la importancia de generar indicadores positivos del bienestar del adolescente que permitan evaluar la calidad del desarrollo a futuro y discute propuestas de acción enmarcadas en un enfoque comunitario del desarrollo juvenil.
\end{abstract}

Palabras Clave: adolescencia, desarrollo juvenil, competencia, contexto evolutivo.

\begin{abstract}
Adolescent development results not only from the strengths and vulnerabilities of the individual adolescent but from the opportunities available in the contexts where adolescents interact daily. This article broadens the understanding of adolescent development by focusing on the role and opportunities that daily life contexts afford to promote competencies and skills in adolescents. The article addresses two challenges for social policy, namely social inequality and the enhancement of social capital. It argues for a positive view of adolescence that focuses on the promotion of competence rather than at-risk behaviors. The need to develop positive indicators of adolescent development and well-being is stressed and guidelines for action are discussed.
\end{abstract}

Keywords: adolescence, youth development, competence, developmental context.

Como etapa vital, la adolescencia plantea nuevos desafíos para el desarrollo humano y la promoción del bienestar. Tanto las tareas evolutivas esperadas, como el proceso de apoyo para el desarrollo positivo de los adolescentes deben comprenderse en el contexto sociocultural en que éstos se desarrollan. Importantes cambios sociales y económicos en el mundo han prolongado la adolescencia (Arnett, 2000) y cambiado el perfil de las tareas evolutivas de esta etapa, planteando desa-

\footnotetext{
M. Loreto Martínez G., Escuela de Psicología, Pontificia Universidad Católica de Chile.

La correspondencia relacionada con este artículo debe ser enviada a M. Loreto Martínez G., Escuela de Psicología, Pontificia Universidad Católica de Chile, Avenida Vicuña Mackenna 4860, Macul, Santiago, Chile. E-mail: mlmartig@uc.cl

La preparación de este artículo fue posible gracias al financiamiento del Fondo Nacional de Desarrollo Científico y Tecnológico (FONDECYT) a través del Proyecto $\mathrm{N}^{\mathrm{o}} 1010934$ "Mirando hacia el futuro: Un estudio longitudinal de los factores que promueven el desarrollo y bienestar en los jóvenes".
}

fíos para la investigación y el diseño de políticas dirigidas a los jóvenes.

Durante los últimos años ha aumentado el interés por identificar las condiciones que favorecen el desarrollo saludable de los adolescentes (Carnegie Council on Adolescent Development, 1989; Moore \& Halle, 2000; Roth \& Brooks-Gunn, 2000) y cómo facilitar este proceso a través de la formulación de políticas sociales y programas de intervención. El énfasis en la promoción de competencias para la adaptación hace evidente que aún los adolescentes que crezcan "sin problemas" pueden no estar bien preparados para enfrentar los desafíos del futuro. Esto es, cuando enfrenten las múltiples opciones para la toma de decisiones en ámbitos de elección de carrera, transición al trabajo, sustento económico y formación de su familia, entre otras. En tiempos actuales, la individualización de la responsabilidad por el bienestar (Programa de las Naciones Unidas para el Desarrollo [PNUD], 2002, 2003) aumenta las libertades para la toma de decisiones, pero también aumenta los riesgos para el/la adolescente (Bendit, 2000). 
El desarrollo positivo incluye la promoción de comportamientos adaptativos y competencias, como también la reducción de comportamientos y actitudes negativas. Este proceso puede favorecerse a través de las oportunidades y los sistemas de apoyo social que existen en las familias, colegios, grupos de pares, lugares de trabajo, organizaciones comunitarias, y otros espacios de interacción. Este artículo presenta un marco conceptual y empírico para la comprensión y promoción del desarrollo positivo de los adolescentes, que enfatiza la importancia del contexto social e identifica factores a nivel del individuo, de la familia, el colegio, la comunidad, y la cultura. Esta discusión puede estimular el desarrollo de investigación, como también la formulación de políticas y programas para promover el desarrollo de los adolescentes.

\section{¿Por qué Invertir en el Desarrollo de los Adolescentes?}

El paso de la niñez a la adolescencia es una de las transiciones evolutivas más trascendentales del ciclo vital (Petersen, Leffert, Graham, Alwin \& Ding, 1997). Los cambios evolutivos (e.g., cambios físicos de la pubertad, expansión del pensamiento, creciente capacidad de exploración y de auto-descubrimiento, logro de la autonomía) conectan a los niños con sus sí-mismos adultos transformados en el plano físico, cognitivo y social (Graber, Brooks-Gunn \& Petersen, 1996). Socialmente, cada transición requiere un cierto cambio de rol, en cómo los adolescentes se ven a sí-mismos (e.g., su autoconcepto, su identidad), cómo ven al mundo (e.g., sus actitudes, sus valores) y cómo son vistos por otras personas.

Por lo anterior, la adolescencia es una etapa de hacer opciones y de tomar decisiones (Erikson, 1968) y conlleva tanto oportunidades (e.g., acrecentamiento de habilidades, asunción de nuevos roles en el trabajo, posición de liderazgo en el liceo) como riesgos (e.g., accidentes, embarazo no planificado, conductas antisociales, conflictos con la ley). Los riesgos y oportunidades no surgen al azar, sino de las experiencias previas en la historia de vida de los adolescentes, y también del ciclo evolutivo de sus respectivas familias. Los adolescentes de sectores económicamente más aventajados reciben mayor patrocinio para su desarrollo y más apoyo social que sus pares de sectores más pobres (Furstenberg, 2001). Las ventajas y desventajas que los adolescentes y sus familias acumulan a través del tiempo les aseguran un cierto grado de continuidad en su desarrollo personal (Furstenberg, Cook, Eccles, Elder \& Sameroff, 1999).

Muchos jóvenes pueden mostrar indicadores temporales de adaptación pero, dependiendo de sus condiciones de vida, no podrán mantener la adaptación en el tiempo, cuando cambien las demandas ambientales (e.g., egreso de enseñanza media, ingreso al trabajo). Dada la precariedad de las circunstancias sociales y familiares de muchos preadolescentes de sectores económicamente desventajados, se hace imprescindible seguir sus pasos, conocer los riesgos que enfrentan y desarrollar programas que apoyen, desde la temprana hasta la tardía adolescencia, los momentos críticos en la toma de decisiones (e.g., la elección de carrera, la mantención de un estilo de vida saludable, el ingreso al trabajo, formación de una familia, todas tareas que los conectan con la adultez). Los estudios de diseño longitudinal permiten documentar el proceso de cambio a través del tiempo tanto en los adolescentes como en sus contextos de vida, identificar condiciones protectoras del desarrollo, las estrategias que padres y adolescentes utilizan para mantener los resultados positivos, y ante qué situaciones emergen.

\section{Tareas y Trayectorias Evolutivas: Oportunidad y Riesgo}

Las tareas evolutivas de la adolescencia, principalmente la configuración de la identidad, el logro de autonomía, y la intimidad, incrementan los ámbitos de elección y decisión de los adolescentes. Así aprenden a auto-dirigirse y a regular su comportamiento hacia la consecución de metas significativas.

A medida que crecen, los adolescentes son más activos en seleccionar los ambientes en los que participan, y las decisiones que toman respecto de su comportamiento y sus metas a futuro. Las elecciones que hacen en estos ámbitos tienen consecuencias para su vida posterior. Tales elecciones o decisiones los comprometen con ciertos cursos de acción y afectan los ambientes sociales en los cuales los adolescentes se insertan (e.g., a través de las interacciones que mantienen en estos contextos), y las influencias que reciben de los pares y adultos con quienes interactúan en estos contextos (Crockett \& Crouter, 1995). Las elecciones o decisiones a corto plazo de un adolescente pueden evolucionar hacia patrones más estables de comportamiento o estilos de vida, los que a su vez, influirán en la calidad de su desarrollo futuro. 
Como período de cambio, la adolescencia ilustra tanto procesos de plasticidad como de consolidación de ciertos patrones de comportamiento. Los cambios biológicos, cognitivos y sociales conllevan oportunidades para el crecimiento, tales como nuevas relaciones sociales, nuevos intereses y nuevos comportamientos. Al mismo tiempo, las disposiciones conductuales que el adolescente trae desde la niñez pueden consolidarse en patrones duraderos de comportamiento a través de dos procesos que favorecen la continuidad del desarrollo, la selección de ambientes y la producción de ambientes (Crockett \& Crouter, 1995). En el primer caso, la mayor autonomía permite al adolescente seleccionar compañía, amistades y ambientes sociales compatibles con sus propios intereses e inclinaciones. En el segundo, debido a su creciente madurez física, social y cognitiva, los adolescentes tienen mayor capacidad de influir en los ambientes en los cuales interactúan (e.g., su grupo de pares, su curso, sus proyectos).

Las decisiones que toma el adolescente van a mostrar cierta continuidad y van a fijar los parámetros de su desarrollo futuro. La acumulación de ciertas decisiones (e.g., practicar un deporte, aprender una habilidad) hace que ciertos resultados evolutivos sean menos probables (e.g., aburrimiento, subir de peso). Pero también, los adolescentes pueden tomar decisiones que ponen en riesgo su vida. Este riesgo parece mayor en la actualidad con la creciente complejización de la vida y el aumento del rango de opciones de decisión (Bendit, 2000). Al mismo tiempo, la desarticulación de los sistemas naturales de apoyo social (e.g., la familia, el barrio) ofrece menor protección frente a las consecuencias negativas de decisiones.

Tanto los cambios normativos (e.g., pubertad), como los no-normativos (e.g., accidente, muerte de un progenitor), e incluso eventos de azar pueden precipitar momentos de cambio en la adolescencia. Para muchos jóvenes, la adolescencia ofrece la oportunidad de encaminarse hacia una vida competente y productiva. Para otros, representa la última oportunidad de prevenir un futuro negativo (Carnegie Council on Adolescent Development, 1989). En ambos casos, los programas de promoción de la salud y bienestar que fortalecen las disposiciones conductuales positivas de los adolescentes tienen beneficios duraderos (Maddaleno, Morello \& Infante-Espínola, 2003; Resnick, 2000).

\section{La Importancia de los Contextos de Desarrollo}

La adolescencia está moldeada por el contexto sociocultural en que se desarrollan los jóvenes. En gran medida, las tareas y las experiencias reflejan la estructuración cultural que hace la sociedad de la adolescencia como una etapa preparatoria de la adultez. Las habilidades que como sociedad esperamos que los adolescentes dominen, las opciones o decisiones que esperamos que tomen y los entornos en que se socializan, están prescritos por las instituciones sociales y por las expectativas culturales compartidas respecto de cuáles son los requerimientos de éxito en la edad adulta (Crockett \& Crouter, 1995). Los adolescentes perciben las expectativas sociales y se proponen metas y tareas basadas en éstas; con frecuencia sus metas personales reflejan tareas evolutivas definidas culturalmente (Nurmi, 1989).

El bienestar de los adolescentes no es resultado únicamente de las fortalezas y vulnerabilidades de cada adolescente, sino también de las oportunidades y la calidad de las experiencias que tienen en los entornos (e.g., familia, colegio, barrio, programas o grupos juveniles) en que interactúan a diario. Estos contextos ofrecen oportunidades y recursos que contribuyen al desarrollo. Por ejemplo, a través de la participación en el centro de alumnos un adolescente puede acceder a otras posiciones de liderazgo, que no sólo fortalecerán sus habilidades sociales si no que, en el contacto con otros grupos y personas, pueden abrir futuras oportunidades vocacionales o laborales.

Siguiendo los postulados de Bronfenbrenner, se ha reconocido que el desarrollo humano está moldeado por el contexto (Bronfenbrenner \& Morris, 1998; Lerner et al., 1996). El desarrollo ocurre a medida que los adolescentes interactúan en los diferentes entornos de su mundo social (e.g., pares, familias, comunidad). El desarrollo se produce a través de los procesos próximos que ocurren dentro de los contextos sociales inmediatos (Bronfenbrenner \& Morris, 1998), procesos que también reciben influencias de otros entornos más distantes (e.g., trabajo de los padres) de la vida cotidiana de los adolescentes.

Si bien la importancia de diferentes niveles de la ecología social para el desarrollo de los adolescentes ha sido enfatizada por varios investigadores (Blyth \& Leffert, 1995; Lerner \& Galambos, 1998), su rol en la promoción del bienestar y la salud del adolescente aún no está bien estudiado. Los roles y relaciones que se establecen en estos entornos for- 
man la base de las interacciones diarias del adolescente con otros significativos en su ambiente social. Posibles mecanismos, a través de los cuales las experiencias en estos contextos promueven el desarrollo, son influyendo en cómo los adolescentes se sienten respecto de sí mismos, afectando las decisiones que toman (lo que a su vez puede afectar sus opciones a futuro); afectando la conducta de los padres; y/o moldeando la estructura de oportunidades disponible para ellos y/o sus familias.

Se requieren estudios empíricos que examinen las oportunidades que ofrecen los entornos y las instituciones, y las maneras en las cuales los adolescentes se conectan a estos ambientes sociales para comprender de qué manera influyen sobre sus competencias y habilidades. Este conocimiento informará el diseño de políticas sociales y de intervenciones dirigidas a fortalecer las oportunidades en entornos cotidianos (e.g., colegio) para que los jóvenes contribuyan a sus comunidades y al país, particularmente en jóvenes de sectores socioeconómicos menos aventajados.

Dos desafíos que enfrenta la formulación de políticas y el diseño de programas para los adolescentes son atender a la heterogeneidad de realidades juveniles (e.g., por condiciones de género, etnia y nivel socioeconómico), como también fortalecer el capital social para la integración de diversos grupos de jóvenes.

\section{Inequidad de Oportunidades}

Como resultado de políticas sociales dirigidas a superar la pobreza y reformar el sistema educacional (Cox, 1998), ha aumentado el acceso a la educación, los años de permanencia en el sistema escolar, las tasas de matrícula y la asistencia a la escuela (Ministerio de Planificación y Cooperación Social [MIDEPLAN], 2003). La cobertura del sistema escolar aumentó un $12.3 \%$ entre 1990 y 2003 en la enseñanza media alcanzando un $92.6 \%$ en el grupo de 14-17 años (MIDEPLAN, 2003). El acceso a educación superior alcanzó a 33\% de los jóvenes entre 19 y 24 años (Instituto Nacional de la Juventud [INJUV], 2004). El nivel educacional de los adolescentes y adultos jóvenes (15-24 años) aumentó de 10.2 años en 1990 a 11.2 años en 2004, y estos logros son más significativos para los jóvenes más pobres (en promedio tienen 1.5 años de educación más que sus padres y 2.5 años más que sus abuelos) y de sectores rurales (en promedio tienen 1.6 años de educación más que sus padres y 2.8 años más que sus abuelos) (MIDEPLAN, 2003).
Otros programas gubernamentales (e.g., Chile Joven, FOSIS, MECE) incluyeron entre sus metas la participación como uno de los mecanismos para desarrollar habilidades y competencias, y así fortalecer la inclusión social de jóvenes de sectores desventajados económicamente. Los indicadores de desarrollo humano en salud, educación y expectativas de vida se encuentran dentro de los mejores de Latinoamérica (Raczynski, 2000), y los indicadores macroeconómicos del país describen un escenario optimista para el futuro desarrollo de los jóvenes chilenos (MIDEPLAN, 1999).

Si bien los ideales democráticos promueven la igualdad de oportunidades, el 19\% de la población adolescente que vive en pobreza, y el $6.6 \%$ que vive en extrema pobreza (MIDEPLAN, 2003) enfrentan limitantes -sociales, económicas y culturales, tanto a nivel individual como colectivo- para su óptimo crecimiento y desarrollo (INJUV, 1999). A nivel institucional, el progreso de los jóvenes en el sistema educacional reproduce la inequidad del sistema social y las deficiencias en la calidad de la educación (Hopenhayn, 2004).

A medida que aumenta la edad los resultados educacionales decrecen (Muñoz, 2000). Los estudiantes no sólo experimentan una alta tasa de repetición de curso, sino que también arrastran deficiencias críticas en su aprendizaje desde la enseñanza primaria que limitan las trayectorias laborales y de vida de los jóvenes (Hopenhayn, 2004). Adicionalmente, en el acceso a tecnología y el dominio de habilidades computacionales básicas se observan amplias diferencias por nivel socioeconómico (INJUV, 2003).

También existen menos oportunidades para jóvenes de sectores pobres, rurales y de hogares cuyos jefes de familia son mujeres. Concordante con el escenario anterior, los jóvenes perciben que el sistema educacional es competitivo, poco facilitador de su desarrollo, y que les entrega una formación que no les permite desenvolverse adecuadamente en el mundo del trabajo (INJUV, 1999).

A nivel familiar, el bajo ingreso económico y las condiciones asociadas a pobreza (e.g., mala calidad de vivienda, hacinamiento, desempleo) como también factores del contexto escolar (e.g., baja calidad del establecimiento educacional) afectan negativamente los resultados educacionales de los jóvenes (Muñoz, 2000). El nivel educacional de los padres, el valor que éstos asignan a la educación y las aspiraciones y prácticas parentales también influyen en las decisiones educacionales de los hijos (Arancibia, 1999; Muñoz, 2000). En este contexto, la percepción 
de falta de posibilidades para desarrollar planes a futuro es real para ciertos grupos de jóvenes, particularmente aquellos con menos educación formal y con responsabilidades familiares (INJUV, 1999).

En otra tarea evolutiva importante como es la transición al trabajo operan fuertes procesos de selección. El criterio de selección que impone la educación formal deja a muchos jóvenes fuera de los mejores trabajos (e.g., con contrato indefinido), engrosando las filas del trabajo desregulado o por cuenta propia (Fernández, 2004). Las condiciones de inestabilidad por ausencia de contrato, trabajos informales e inestables afectan primordialmente a los jóvenes menores de 20 años (INJUV, 2004).

Se observa también una concentración del empleo juvenil en sectores de baja productividad y las mujeres muestran condiciones más desfavorables de inserción al trabajo que sus pares masculinos (Hopenhayn, 2004). Lo anterior contrasta con el mayor logro educacional y las crecientes aspiraciones de las jóvenes. Un porcentaje mayor (65.9\%) de mujeres jóvenes aspira a obtener un grado universitario comparado con sus pares hombres (56.9\%), cifras que señalan cambio en las expectativas asociadas al rol en relación a generaciones anteriores (Servicio Nacional de la Mujer [SERNAM], 2002) y crearán tensiones entre aspiraciones $\mathrm{y}$ oportunidades que afectarán las trayectorias de vida de estas jóvenes, no sólo en el ámbito educacional y laboral, sino en la entrada a otros roles sociales (e.g., madre, esposa).

Las diferencias por género y nivel socioeconómico requieren ser examinadas en detalle para generar políticas sociales que respondan a las necesidades reales de diferentes grupos de jóvenes. Por ejemplo, el inicio de la paternidad antes de los 20 años es un factor que atenta contra la educación completa de un porcentaje sustantivo de jóvenes de nivel socioeconómico bajo. Alternativamente, la mayoría de los jóvenes de nivel socioeconómico alto decide iniciar la paternidad más tarde (INJUV, 1999). Estas observaciones muestran diferencias culturales por nivel socioeconómico, y su ingerencia en las decisiones de los jóvenes debe ser tomada en cuenta al momento de planificar programas de promoción del desarrollo y/o políticas sociales.

Al comparar los resultados de encuestas nacionales de juventud, se observa que el porcentaje que abandona la enseñanza media para empezar a trabajar aumenta de $14.1 \%$ en 1994 a $25.45 \%$ en 1997 (INJUV, 1999). El aumento del porcentaje de jóvenes que decide comenzar a trabajar y desconti- nuar su educación puede reflejar la falta de prospectos de mejoría laboral como consecuencia de su permanencia en el sistema escolar y/o la maximización del ingreso como vía efectiva hacia la autonomía (Espinoza, 1999). Desafortunadamente, la naturaleza transversal de las encuestas nacionales de juventud no permite analizar los procesos (e.g., a nivel personal, familiar y de la comunidad) que inciden en las decisiones de los jóvenes y cómo éstas pueden moderar los efectos de barreras económicas y sociales en su desarrollo. Este conocimiento es central para la formulación de políticas sociales efectivas que respondan a las necesidades de jóvenes de diferente género y condiciones socioe-conómicas y culturales.

\section{Fortalecimiento del Capital Social}

Además de los recursos personales, otros estudios destacan la importancia de la cualidad de la inserción de la familia en la comunidad para el desarrollo de niños (Martínez, 2000) y jóvenes (Sampson, Raudenbusch \& Earls, 1997). Algunos aspectos de la organización social de la comunidad, tales como el grado en que otros adultos, además de los padres, intervienen en la supervisión de los adolescentes, y guían su comportamiento hacia normas prosociales, la densidad de las redes sociales (Crittenden, 1985), y los niveles de participación en organizaciones comunitarias previenen la desviación social en los jóvenes (Sampson \& Groves, 1989).

Coleman (1988) usa el término "capital social" para referirse a los recursos comunitarios (e.g., normas, obligaciones mutuas, sentido de comunidad, y oportunidades para compartir información) que promueven el desarrollo de niños y adolescentes. De acuerdo a esta perspectiva, la comunidad puede ser entendida como una reserva de capital social puesto que sus redes de relaciones y organizaciones sociales (tanto formales como informales) aportan recursos a los padres para lograr las metas de socialización que se han propuesto con sus hijos.

Es responsabilidad pública crear y apoyar los caminos para que los jóvenes transiten hacia su integración social exitosa y generar las condiciones para que se conviertan en ciudadanos productivos y creativos (INJUV, 1999). Los jóvenes de sectores económicos menos privilegiados (e.g., mapuches, de nivel socioeconómico bajo) están expuestos a desventajas socioculturales y económicas que debilitan la estructura de oportunidades para una transición favorable a la adultez, y/o pueden presentar 
riesgos para una adaptación efectiva. Además de incrementar la inversión en educación, es necesario identificar los recursos disponibles en la comunidad que puedan fortalecer los esfuerzos iniciados en la familia y en el sector educacional para desarrollar el capital humano de los jóvenes.

La comprensión de las influencias del nuevo contexto social y cultural es central para la formulación de políticas de desarrollo humano dirigidas a jóvenes. En un mundo de economía globalizada, la educación y el fortalecimiento del capital humano son requisitos indispensables para el desarrollo social y económico de los países. Los cambios tecnológicos, organizativos y las presiones por la competitividad, demandan nuevas capacidades a la fuerza de trabajo. La formación de los jóvenes en el uso de nuevas tecnologías debe considerar también nuevas habilidades personales, sociales, e intelectuales necesarias para su adaptabilidad eficiente a los cambios en el mundo laboral (Fernández, 2004).

Como ciudadanos, los jóvenes proyectarán el desarrollo que ha alcanzado el país, y la inversión en ellos creará vías para la sustentación de la democracia. A nivel nacional, existe mayor conciencia de la necesidad de incluir a amplios sectores de jóvenes para que ejerzan plenamente su ciudadanía y mejoren su calidad de vida, ideal expresado en la misión del Instituto Nacional de la Juventud, creado en 1991. Si bien se ha avanzado en definir las bases para una política de juventud que mejore la gestión y ejecución de programas y el diseño de servicios, la formulación de esta política -entendida como un conjunto de principios, metas y recursos para implementar objetivos de desarrollo- es una tarea pendiente, que necesariamente requiere de conocimientos acerca de las aspiraciones, necesidades y competencias de los jóvenes.

Capitalizando en los resultados favorables de políticas sociales para combatir la pobreza, la reforma educacional y los programas de capacitación juvenil, el periodo actual ofrece una oportunidad única de fortalecer la investigación que pueda informar la formulación eficiente de programas y políticas sociales para fortalecer el desarrollo de competencias y la integración social de la juventud. La identificación de factores protectores del desarrollo a nivel de la familia, la participación comunitaria, y la comprensión de los mecanismos a través de los cuales éstos operan, constituyen los fundamentos sobre los cuales se diseñan, planifican y evalúan políticas sociales dirigidas a jóvenes, familias y comunidades.

\section{Visión Positiva de la Adolescencia}

Como educadores, padres, profesionales o diseñadores de políticas sociales aspiramos a que los adolescentes alcancen logros que van más allá de la evitación de ciertos riesgos, pero disponemos de poca literatura sobre las condiciones que promueven el desarrollo positivo en los adolescentes. Los temas de investigación, los programas de intervención y las imágenes difundidas en medios de comunicación se centran frecuentemente en comportamientos "problema" de los jóvenes. Alternativamente, se puede enfatizar el conocimiento de los aspectos positivos del desarrollo adolescente, habilidades y competencias, y las maneras a través de las cuales los jóvenes negocian constructivamente las transiciones evolutivas para convertirse en adultos competentes y productivos para el país. Diversos estudios (Benson, Leffert, Scales \& Blyth, 1998; Jessor, Turbin \& Costa, 1998; Leffert et al., 1998; Scales, Benson, Leffert \& Blyth, 2000) documentan la relación entre los recursos adaptativos y comportamientos de riesgo, como también los resultados positivos.

\section{Competencia Social}

El concepto de competencia social ha recibido atención en el estudio del funcionamiento adolescente por su potencial para explicar la adaptación positiva (Cowen, 1994; Masten et al., 1995). Competencia denota la capacidad de la persona para enfrentar las demandas ambientales y aprovechar las oportunidades de manera activa y efectiva. La competencia se construye a través de experiencias y oportunidades para involucrarse en actividades, proyectos de interés, y/o en la relación con pares o adultos significativos con los cuales los adolescentes interactúan. Estos esfuerzos se caracterizan por ser mantenidos en el tiempo (Larson, 2000) y pueden promoverse a través de programas desarrollados en contextos formales (e.g., colegio) o informales (e.g., grupos de juventud) (Larson \& Verma, 1999).

Si bien se reconoce la importancia de estudiar los recursos para la adaptación y las competencias de las personas (Larson, 2000; Masterpasqua, 1989), aún existe muy poco énfasis, tanto en la investigación como en las políticas sociales, en cómo promover el desarrollo positivo en los adolescentes. Con excepción de las encuestas nacionales (INJUV, 1999, 2004) y algunos estudios empíricos (Maddaleno et al., 2003), el grupo adolescente ha sido descuidado 
como objetivo de investigación y de políticas sociales en nuestro país.

La falta de evidencia de investigación sobre los aspectos positivos del desarrollo se debe también a falta de consenso entre expertos acerca de cuáles son los resultados deseables para los adolescentes. Esta carencia impide dar una clara orientación a padres, educadores, líderes juveniles y comunitarios sobre las metas a lograr con los jóvenes. Tanto investigadores como expertos en políticas sociales requieren concertar esfuerzos para desarrollar indicadores que reflejen la diversidad de competencias definidas de acuerdo a las normas culturales de diferentes grupos de juventud (Espinoza, 1999; Moore \& Halle, 2000).

En respuesta a imágenes negativas de los jóvenes, los programas de promoción del bienestar a menudo se centran en prevenir comportamientos de riesgo. La perspectiva centrada en los riesgos es limitada, puede sobreestimar la frecuencia de comportamientos negativos y subestimar la participación de los jóvenes en actividades positivas y productivas que los encamina en trayectorias de competencia. Por ejemplo, los hallazgos de una muestra nacional de juventud (INJUV, 2004) indican que cerca del 48\% de los jóvenes (entre 15-29 años) participa en organizaciones, principalmente deportivas, religiosas y virtuales, siendo mayor este porcentaje en los adolescentes jóvenes (entre 15-18 años). Asimismo, las crecientes cifras de enrolamiento de jóvenes en programas de ayuda social y en iniciativas de emprendimiento solidario también dan cuenta de motivaciones y compromisos de los jóvenes con causas sociales.

La movilización estudiantil del 2006 en demanda de una mejor calidad de la educación en Chile ilustra manifestaciones de ciudadanía desde la perspectiva de los jóvenes y desafía la visión de una juventud apática. El apoyo que concitó este movimiento en amplios sectores de juventud revela la importancia de atender a los asuntos que definen las expresiones ciudadanas en los jóvenes. Los jóvenes valoran la democracia, afirman que ésta debe ser perfeccionada y son críticos de la sociedad chilena por su inequidad y falta de oportunidades (INJUV, 2002). El $47 \%$ de ellos piensa que no hay igualdad en el acceso a oportunidades; el $35 \%$ cree que debieran reducirse las diferencias de clase y la inequidad social, y el 29\% demanda justicia social (INJUV, 2004). Un grupo importante de jóvenes refiere que se ha sentido discriminado por clase social, edad o por su condición de estudiante (INJUV, 2004). En síntesis, los jóvenes poseen expectativas acerca de sus derechos como ciudadanos e identifican barreras para el ejercicio de éstos. Por tanto, es necesario crear las oportunidades para que se involucren como actores de su desarrollo.

Otros indicadores positivos se encuentran en la construcción de la identidad personal, considerada un importante medio para la consecución de las metas de vida. Los adolescentes chilenos (15-18 años) tienen autoconcepto positivo y alta autoestima. Se describen a sí mismos con atributos positivos tales como solidaridad (36.9\%), sociabilidad (34.0\%), orientados a la realidad (28.7\%), flexibles $(28.6 \%)$ y optimistas (25.4\%) (INJUV, 2004).

Otros hallazgos de muestras nacionales (INJUV, 1999, 2004; PNUD, 2003) sobre las aspiraciones y los valores de la juventud chilena destacan los recursos y fortalezas que ésta posee para enfrentar su desarrollo futuro. Los jóvenes muestran una alta disposición a la integración social, valoran la educación formal y el trabajo como el camino para alcanzar esta integración (Espinoza, 1999), y la formación de sus propias familias. En síntesis, se descartan las imágenes negativas de la juventud difundidas durantes los años 80, que enfatizaban la exclusión, la desintegración cultural, y las conductas conflictivas de la juventud (INJUV, 1999).

Los adolescentes chilenos refieren altas aspiraciones y expectativas de logro educacional futuro (INJUV, 2002; Martínez, Cumsille \& Rivera, 2004; PNUD, 2003), particularmente en mujeres. Martínez, Cumsille y Rivera (2004) observaron que estas aspiraciones y expectativas disminuyen a medida que los adolescentes crecen, hallazgo que probablemente refleja una autoevaluación más realista de sus capacidades. Sin embargo, el nivel socioeconómico se asocia positivamente con las aspiraciones y expectativas educacionales, probablemente asociado a influencias de la familia y del sistema escolar. Esta observación destaca la importancia de acrecentar las oportunidades y disminuir las barreras para el logro de los adolescentes de sectores económicamente desventajados, y de atender diferencialmente a las expectativas de las adolescentes (Martínez, Cumsille \& Rivera, 2004), en consonancia con cambios en las expectativas de rol para las mujeres en Chile (PNUD, 2003).

\section{Participación Social y Desarrollo Cívico}

Diversos estudios (Flanagan, Bowes, Jonsson, Csapo \& Sheblanova, 1998; Flanagan \& Gill, 1999) 
sugieren que las experiencias de pertenencia y la participación en grupos e instituciones de la comunidad (e.g., organizaciones escolares o juveniles, culturales, ecologistas, políticas, comunidades religiosas) promueve la integración social y protege a los jóvenes de conductas negativas. Paralelamente, la salud de los sistemas sociales (e.g., familias, colegios, comunidades) depende de la participación activa y del compromiso de sus miembros, procesos que desarrollan confianza social y sentido del bien común (Flanagan \& Gill, 1999).

Los hallazgos de las encuestas nacionales de juventud sugieren que las tendencias de participación juvenil por estrato socioeconómico han cambiado desde 1994 a 1997. La participación en organizaciones sociales y políticas de los jóvenes ha disminuido, fenómeno descrito como "desapego político" (Espinoza, 1999), que también se ha observado en otras sociedades durante períodos de cambio social rápido (Flanagan et al., 1998). Estas conclusiones deben interpretarse con cautela. La naturaleza transversal de los datos INJUV, como también la agregación de jóvenes en un intervalo amplio de edad (e.g., 15 a 29 años) impide analizar la evolución de estos comportamientos en cohortes de jóvenes durante un período histórico particular y se hace necesario el diseño de estudios longitudinales.

Sabemos muy poco acerca de los procesos a través de los cuales los jóvenes se convierten en buenos ciudadanos (e.g., cuáles son las vías de inclusión), y mucho menos acerca de cómo estos procesos ocurren en jóvenes de grupos económicamente desventajados o históricamente marginalizados, y si difieren de los procesos que experimentan jóvenes de sectores más afluentes. La competencia cívica supone el desarrollo de habilidades prosociales, el apego positivo a instituciones sociales (e.g., familia, colegio), y la adhesión a valores de responsabilidad social, respeto por la diversidad, los principios democráticos y los derechos civiles.

Es central conocer los factores que contribuyen a que los jóvenes aprendan y se motiven por participar en la vida cívica de sus comunidades, y de cómo estas experiencias a nivel local pueden crear oportunidades para la participación formal de los jóvenes en la vida cívica del país (Espinoza, 1999). En una muestra de 1093 adolescentes, Silva y Martínez (en prensa) encontraron que la participación en actividades escolares como debates, centro de alumnos, promueve la eficacia política y la conciencia crítica de los jóvenes.
Los colegios son contextos claves para aprender y practicar la ciudadanía y los principios democráticos, sin embargo, se requiere desarrollar y evaluar iniciativas para llevar a la práctica los principios de equidad, solidaridad y tolerancia a la diversidad. Algunas intervenciones han mostrado resultados favorables pero requieren ser replicadas y/o evaluadas en otros grupos de jóvenes. Por ejemplo, utilizando una metodología de investigación participativa, Prieto (2003) encontró que los jóvenes no sólo mejoraron su conocimiento acerca de la democracia, sino que aumentaron el conocimiento de sí mismos, aprendieron a expresar sus opiniones, y a monitorear su comportamiento tomando en cuenta los sentimientos y las acciones de otros.

\section{Actividades Significativas y Uso del Tiempo Libre}

Las actividades instrumentales son un componente diario de la vida de los adolescentes (Larson, 2000), y un medio para la exploración de la identidad. Las actividades incluyen pasar tiempo en otros contextos tales como el trabajo, los grupos de amigos, proyectos juveniles, y organizaciones comunitarias. Estas actividades implican interacciones regulares con adultos (e.g., entrenadores, mentores, líderes) y ayudan a formar el autoconcepto, desarrollan la iniciativa personal, intereses, y desalientan las conductas problema (Larson, 2000). Además de crear lazos intergeneracionales, las figuras adultas pueden servir de modelos, dar apoyo social y canalizar el comportamiento de los adolescentes hacia metas deseadas.

Los hallazgos de una muestra nacional de juventud (INJUV, 2004) indican que los jóvenes chilenos no están participando en actividades significativas para su desarrollo. El fútbol da cuenta de la mayor participación juvenil en actividades organizadas, particularmente en varones. Los beneficios de esta participación no están claros pues también se asocia con consumo de alcohol y drogas (INJUV, 1999). En una muestra de jóvenes urbanos de nivel socioeconómico bajo, Martínez y Cumsille (1996) encontraron altos niveles de sintomatología depresiva, tales como aburrimiento frecuente, pesimismo y soledad. Estos índices de alienación y falta de perspectiva a futuro en una población de jóvenes que puede describirse como "normal", no constituye necesariamente psicopatología, y pueden reflejar falta de proyectos que los motiven intrínsecamente o que los dirijan a la consecución de metas importantes para ellos (Larson, 2000). Futuros estudios podrán investigar que características de las actividades diarias - estructuradas y no estructuradas- y de 
los contextos en que éstas se realizan, contribuyen al desarrollo de iniciativas y proyectos a futuro en los jóvenes.

\section{Sugerencias Para Futuros Estudios y Propuestas Para la acción}

\section{Necesidad de Estudios Longitudinales}

Los hallazgos de las sucesivas encuestas nacionales de juventud (INJUV, 1999, 2002, 2004) aportan importantes descripciones de la realidad juvenil. Sin embargo, para avanzar en el diseño de intervenciones de promoción o prevención, necesitamos comprender de qué manera los factores demográficos a nivel familiar, escolar y de la comunidad interactúan con procesos personales y familiares para producir resultados positivos en los adolescentes. En Chile, la investigación sobre los factores que contribuyen al desarrollo positivo de los jóvenes carece de datos longitudinales que permitan identificar los procesos y mecanismos que contribuyen a que los jóvenes mantengan trayectorias positivas en su desarrollo, aun en presencia de condiciones de riesgo ambiental o desventajas socioeconómicas y culturales.

\section{Énfasis en las Competencias}

La noción de adolescencia de Stanley Hall como un período necesariamente conflictivo llevó a prestar poca atención a las competencias, los recursos, y las actividades prosociales de los adolescentes. Alternativamente, los modelos que se enfocan al desarrollo positivo (e.g., enfatizan habilidades, competencias, recursos) de los jóvenes debieran ser el objetivo de los esfuerzos comunitarios (Bogenschneider, 1998; Villarruel, Perkins, Borden $\&$ Keith, 2003). Los adolescentes que crecen en comunidades que promueven los aspectos positivos de su desarrollo tienen una mejor comprensión de sus valores, son buenos aprendices de por vida, participan activamente en sus comunidades, y con mayor frecuencia, también promueven el bienestar de otros jóvenes (McLaughlin, 2000).

Las características, tanto individuales como ambientales, que facilitan la transición positiva a la adultez, constituyen las piedras fundantes del desarrollo positivo y han sido denominados recursos evolutivos (Benson et al., 1998), habilidades para la vida (Botvin et al., 2000) y factores protectores (Resnick, 2000). Los recursos evolutivos pueden ser dimensiones externas de los ambientes de vida (e.g., relacio- nes positivas con su familia, apego a la escuela, amistades duraderas), o dimensiones internas que reflejan valores, actitudes, y habilidades personales del/la adolescente. Estos recursos se relacionan negativamente con comportamientos de riesgo. Mientras más recursos (e.g., competencias, habilidades) posea un/ a adolescente, es menos probable que se involucre en comportamientos de riesgo, y más probable que se involucre en comportamientos positivos o saludables (Benson et al., 1998; Jessor et al.,1998; Leffert et al., 1998; Scales et al., 2000).

El desarrollo de programas para jóvenes debe incorporar la creación de recursos, fortaleciendo las condiciones favorables existentes en los ambientes naturales (Bogenschneider, 1998). Por ejemplo, a nivel de colegios se puede promover la participación comunitaria a través del involucramiento de los jóvenes en programas de servicio voluntario (Reinders \& Youniss, 2006). Estos programas pueden adoptar metodologías participativas que posibilite a los adolescentes tomar roles activos en el diseño y evaluación de los mismos.

Los profesionales, investigadores y diseñadores de políticas públicas pueden colaborar con los jóvenes en la planificación de programas y en la movilización comunitaria para crear ambientes en que los jóvenes establezcan relaciones positivas con adultos, y desarrollen competencias y habilidades (Eccles \& Gootman, 2001). Al mismo tiempo, deben investigar la medida en que estos programas son efectivos y constituyen buenos contextos para el desarrollo adolescente, vinculando sus características y recursos con medidas de resultado en los participantes (Larson, 2000). Para avanzar en este objetivo se requiere generar indicadores que documenten resultados positivos en el desarrollo, tales como habilidades sociales y cognitivas, autoconcepto, participación en actividades significativas, pertenencia a grupos positivos con pares, estrategias positivas de afrontamiento y estilos de vida saludables (Jessor et al., 1998; Lerner \& Galambos, 1998).

\section{Adolescentes Como Agentes de su Desarrollo}

El desafío para los programas es comprometer a los jóvenes en procesos que promuevan su propio desarrollo (e.g., liderazgo, iniciativa, emprendimiento), y capacitarlos para tomar las decisiones que les permitan lograr este objetivo. En otras palabras, tanto las políticas como los programas de juventud debieran capitalizar en la capacidad de agencia y en la iniciativa de los jóvenes, de manera 
de comprometerlos a forjar su propio desarrollo (Pittman, 2000). La tarea pendiente es, sin embargo, generar los espacios y procesos, a nivel local y nacional, para incluir la voz y fortalecer el liderazgo juvenil. La juventud de sectores históricamente marginalizados puede requerir atención especial (e.g., a través de programas y políticas) para incluirse en forma creativa y competente, de manera que se conviertan en actores de su desarrollo personal y del de sus comunidades.

\section{Enfoque Comunitario del Desarrollo Adolescente}

Un enfoque comunitario promueve el compromiso de los jóvenes con su propio desarrollo (Larson, 2000) y el de sus comunidades dándoles múltiples oportunidades de aprender roles y competencias, y de establecer conexiones positivas con otros, y con la comunidad (Villarruel et al., 2003; Zeldin, Camino $\&$ Calvert, 2003). Actualmente, los entornos de muchas comunidades o barrios urbanos, no solamente aquellos que concentran mayor pobreza o violencia, necesitan rediseñarse para dar oportunidades a jóvenes de involucrarse en comportamientos positivos, y prevenir conductas de riesgo.

La pregunta a responder es ¿qué características de las comunidades crean ambientes saludables para la promoción del desarrollo y la salud de los adolescentes? A nivel local, es importante identificar y/o fortalecer los sistemas de apoyo social, la estructura de oportunidades, y el rol de las instituciones (oficinas de juventud, centros juveniles) comunitarias para ampliar la inclusión de los jóvenes como agentes activos de sus comunidades. Lo anterior requiere crear modelos de colaboración a diferentes niveles entre organizaciones formales e informales que desarrollan políticas y programas para los jóvenes.

Nuestro conocimiento acerca de cuáles condiciones son importantes de gestar, como también de los procesos a través de los cuales las características comunitarias fortalecen aspectos del desarrollo adolescente es muy limitado, particularmente de estudios que informen acerca de la realidad de nuestro país. El conocimiento de estos factores es indispensable para guiar la oferta de programas. Los psicólogos sociales, comunitarios, y otros profesionales necesitan generar conocimiento acerca de las acciones y características de los programas institucionales e iniciativas de otras organizaciones que efectivamente crean el escenario comunitario que proporciona las oportunidades óptimas para el desarrollo saludable.

\section{Referencias}

Arancibia, V. (1999). Factores que afectan el rendimiento escolar de los pobres. En E. Cohen (Ed.), Educación, deficiencia y equidad (pp. 157-213). Santiago: CEPAL/ OEA/SUR.

Arnett, J. (2000). Emerging adulthood: A theory of development from the late teens through the twenties. American Psychologist, 55(5), 469-480.

Bendit, R. (2000). Participación social y política de los jóvenes en países de la Unión Europea. En S. Balardini (Ed.), La participación social y política de los jóvenes en el horizonte del nuevo siglo (pp. 19-57). Buenos Aires: Clacso.

Benson, P., Leffert, N., Scales, P. \& Blyth, D. (1998). Beyond the "Village" rhetoric: Creating healthy communities for children and adolescents. Applied Developmental Science, 2, 138-159.

Blyth, D. A. \& Leffert, N. (1995). Communities as contexts for adolescent development. Journal of Adolescent Research, 10, 64-87.

Bogenschneider, K. (1998). What youth need to succeed: The roots of resiliency. En K. Bogenschneider \& J. Olson (Eds.), Building resiliency and reducing risk: What youth need from families and communities to succeed (pp. 123). Wisconsin Family Impact Seminars Briefing Report. Madison, WI: Center for Excellence in Family Studies.

Botvin, G. J., Griffin, K. W., Diaz, T., Scheier, L. M., Williams, C. \& Epstein, J. A. (2000). Preventing illicit drug use in adolescents: Long-term follow-up data from a randomized control trial of a school population. Addictive Behaviors, 5, 769-774.

Bronfenbrenner, U. \& Morris, P. A. (1998). The ecology of developmental processes. En W. Damon (Series Ed.) \& R. M. Lerner (Vol. Ed.), Handbook of child psychology: Vol. 1. Theoretical models of human development (5th Ed., pp. 993-1028). New York: Wiley.

Carnegie Council on Adolescent Development. (1989). Turning points: Preparing American youth for the $21^{\text {st }}$ century. New York: Carnegie Corporation.

Coleman, J. (1988). Social capital in the creation of human capital. American Journal of Sociology, 94, 95-120.

Cowen, E. (1994). The enhancement of psychological wellness. American Journal of Community Psychology, 22, 149-179.

Cox, C. (1998). La reforma de la educación chilena en los años 90. Revista Chilena de Humanidades, 18/19, 1347.

Crittenden, P. (1985). Social networks, quality of child rearing, and child development. Child Development, 56, 1299-1313.

Crockett, L. \& Crouter, A. (1995). Pathways through adolescence: Individual development in relation to social contexts. Mahwah, NJ: Erlbaum.

Eccles, J. \& Gootman, J. (2001). Community programs to promote youth development. Washington, DC: National Academy Press.

Erikson, E. (1968). Identidad, juventud y crisis. Buenos Aires: Paidós.

Espinoza, V. (1999, noviembre). Ciudadanía juvenil y politicas públicas. Ponencia presentada en el Seminario Los Jóvenes: Realidades y Tendencias, Instituto Nacional de la Juventud, Santiago, Chile. 
Fernández, P. (2004). Flexibilidad laboral para los jóvenes chilenos. Tesis de Magíster no publicada, Facultad de Ciencias Físicas y Matemáticas, Universidad de Chile, Santiago.

Flanagan, C., Bowes, J., Jonsson, B., Csapo, B. \& Sheblanova, E. (1998). Ties that bind: Correlates of adolescents' civic commitments in seven countries. Journal of Social Issues, 54(3), 454-475.

Flanagan, C. \& Gill, S. (1999, abril). Adolescents' social integration and affection for the polity: Processes for different racial/ethnic groups. Ponencia presentada en Biennial Meeting of the Society for Research in Child Development, Albuquerque, New Mexico.

Furstenberg, F. (2001). Managing to make it. Journal of Family Issues, 22(2), 150-162.

Furstenberg, F., Cook, T., Eccles, J., Elder, G. \& Sameroff, A. (1999). Managing to make it. Chicago: The University of Chicago Press.

Graber, J., Brooks-Gunn, J. \& Petersen, A. (1996). Adolescent transitions in context. En J. Graber, J. Brooks-Gunn \& A. Petersen (Eds.), Transitions through adolescence (pp. 369383). Mahwah, NJ: Erlbaum.

Hopenhayn, M. (2004). La juventud en Iberoamérica: Tendencias y urgencias. Santiago, Chile: Organización Iberoamericana de Juventud CEPAL. Recuperado desde http:// www.eclac.cl/publicaciones/DesarrolloSocial/0/ LCL2180PE/CEPAL_OIJ.pdf

Instituto Nacional de la Juventud. (2004). La integración social de los jóvenes en Chile 1994-2003 (Cuarta Encuesta Nacional de la Juventud). Gobierno de Chile: Ministerio de Planificación y Cooperación.

Instituto Nacional de la Juventud. (2002). La eventualidad de la inclusión. Jóvenes chilenos a comienzos del nuevo siglo (Tercera Encuesta Nacional de la Juventud). Gobierno de Chile: Ministerio de Planificación y Cooperación.

Instituto Nacional de la Juventud. (1999). Sociabilidad y cultura juvenil. Gobierno de Chile: Ministerio de Planificación y Cooperación.

Jessor, R., Turbin, M. S. \& Costa, F. M. (1998). Risk and protection in successful outcomes among disadvantaged adolescents. Applied Developmental Science, 2, 194-208.

Larson, R. (2000). Toward a psychology of positive youth development. American Psychologist, 55(1), 170-183.

Larson, R. \& Verma, S. (1999). How children and adolescents spend time across the world: Work, play and developmental opportunities. Psychological Bulletin, 125(6), 701-736.

Leffert, N., Benson, P., Scales, P., Sharma, A., Drake, D. \& Blyth, D. (1998). Developmental assets: Measurement and prediction of risk behaviors among adolescents. Applied Developmental Science, 2, 209-230.

Lerner, R. \& Galambos, N. (1998). Adolescent development: Challenges and opportunities for research, programs and policies. Annual Review of Psychology, 49, 413-446.

Lerner, R., Lerner, J., von Eye, A., Ostrom, C., Nitz, K., Talwar, R. \& Tubman, J. (1996). Continuity and discontinuity across the transition to early adolescence: A developmental contextual perspective. En J. Graber, J. Brooks-Gunn \& A. Petersen (Eds.), Transitions through adolescence (pp. 3-22). Mahwah, NJ: Erlbaum.

Martínez, M. L. (2000). Neighborhood context and the development of african american children. New York, NY: Garland Publishing.

Martínez, M. L. \& Cumsille, P. (1996). Bienestar psicológico de adolescentes urbanos: Su relación con niveles de competencia psicosocial, sistemas de apoyo social y calidad del tiempo libre. Psykhe, 5, 185-202.
Martínez, M. L., Cumsille, P. \& Rivera, D. (2004, julio). Adolescents' future-oriented aspirations and goals: The role of family expectations and parental practices. Ponencia presentada en $18^{\text {th }}$ Biennial Meeting of the International Society for the Study of Behavioural Development, Gantes, Bélgica.

Martínez, M. L., Cumsille, P. \& Thibaut, C. (2006). Chile. Routledge International Encyclopedia of Adolescence, 2, 167-178.

Maddaleno, M., Morello, P. \& Infante-Espínola, F. (2003). Salud y desarrollo de adolescentes y jóvenes en Latinoamérica y el Caribe: Desafíos para la próxima década. Salud Pública de México, 45(1), 132-139.

Masten, A, Coatsworth, J., Neeman, J., Gest, S., Tellegen, A. \& Garmezy, N. (1995). The structure and coherence of competence from childhood through adolescence. Child Development, 66, 1636-1659.

Masterpasqua, F. (1989). A competence practice for psychological practice. American Psychologist, 44, 1366-1371.

McLaughlin, M. (2000). Community counts: How youth organizations matter for youth development. Washington, DC: Public Education Network. Recuperado desde http://www.PublicEducation.org

Ministerio de Planificación y Cooperación Social. (1999). Reporte económico y social 1990-1996. Santiago, Chile: MIDEPLAN

Ministerio de Planificación y Cooperación Social. (2003). Encuesta de Caracterización Socioeconómica Nacional (CASEN). Santiago: Gobierno de Chile.

Moore, K. \& Halle, T. (2000). Preventing problems versus promoting the positive: What do we want for our children? Washington, DC: Child Trends.

Muñoz, P. (2000, marzo). Socioeconomic and sociodemographic factors associated with the quality of education in Chile: A multilevel exploration. Trabajo presentado en la XXII International Conference of the Latin American Studies Association, Miami, Florida.

Nurmi, J. E. (1989). Development of orientation to the future during early adolescence: A four-year longitudinal study and two cross-sectional comparisons. International Journal of Psychology, 24, 195-214.

Petersen, A., Leffert, N., Graham, B., Alwin, J. \& Ding, S. (1997). Promoting mental health during the transition into adolescence. En J. Schulenberg, J. Maggs \& K. Hurrelman (Eds.), Health risks and developmental transitions during adolescence (pp. 471-497). New York, NY: Cambridge University Press.

Pittman, K. J. (2000). Balancing the equation: Communities supporting youth, youth supporting communities. Community Youth Development Journal, 1, 33-36.

Prieto, M. (2003). Students as agents of democratic renewal in Chile. Forum, 43(2), 87-90.

Programa de las Naciones Unidas para el Desarrollo. (2002). Desarrollo humano en Chile: Nosotros los chilenos: Un desafio cultural. Santiago, Chile: Autor.

Programa de las Naciones Unidas para el Desarrollo. (2003). Transformaciones culturales e identidad juvenil en Chile. Santiago, Chile: Autor.

Raczynski, D. (2000). Chile: Progress, problems, and prospects. En D. Morales-Gómez, N. Tschirgi \& J. Moher (Eds.), Reforming social policy (pp. 45-82). Ottawa, Canada: International Development Research Centre.

Reinders, H. \& Youniss, J. (2006). School-based required community service and civic development in adolescents. Applied Developmental Science, 10(1), 2-12. 
Resnick, M. (2000). Protective factors, resilience, and healthy youth development. Adolescent Medicine, 11(1), 157-164.

Roth, J. \& Brooks-Gunn, J. (2000). What do adolescents need for healthy development? Implications for youth policy. SRCD Social Policy Report, 14(1), 1-19.

Sampson, R. \& Groves, W. (1989). Community structure and crime: Testing social disorganization theory. American Journal of Sociology, 94, 775-802.

Sampson, R., Raudenbusch, S. \& Earls, F. (1997). Neighborhoods and violent crime: A multilevel study of collective efficacy. Science, 277, 918-924.

Scales, P. C., Benson, P. L., Leffert, N. \& Blyth, D. A. (2000). Contribution of developmental assets to the prediction of thriving among adolescents. Applied Developmental Science, 4, 27-46.

Fecha de recepción: Mayo de 2006.

Fecha de aceptación: Diciembre de 2006.
Servicio Nacional de la Mujer. (2002). Análisis y detección de expectativas de vida y proyecto de vida en niños, niñas y adolescentes (Documento de trabajo $\mathrm{N}^{\circ}$ 80). Recuperado desde http://www.sernam.cl/admin/docdescargas/centrodoc.

Silva, C. \& Martínez, M. L. (en prensa). Empoderamiento, participación y autoconcepto de persona socialmente comprometida en adolescentes chilenos. Revista Interamericana de Psicología.

Villarruel, F., Perkins, D., Borden, L. \& Keith, J. (2003). Community youth development. Thousand Oaks, CA: Sage.

Zeldin, S., Camino, L. \& Calvert, M. (2003). Toward an understanding of youth in community governance: Policy priorities and research directions. Social Policy Report, 23(3), 3-20. 